\title{
Effect of L- Carnitine and COQ10 Addition to SMARTPro- Medium on Human SpermConcentration,SpermMorphology and Chromatin StructureduringIn VitroSperm Activation
}

\author{
Majida H.Mutlag Al-Shammari ${ }^{1}$, Jabbar H. Yenzeel ${ }^{1} \&$ \\ Muhammad-Baqir M-R Fakhrildin ${ }^{2}$ \\ ${ }^{1 .}$ Department of Biology, College of Science, University of Baghdad. \\ ${ }^{2 .}$ Dept. Medical Physiology, College of Medicine, Jabir IbnHayyan Medical University/ Iraq.
}

\begin{abstract}
This study was designed to assess the effect of different concentrations of L-carnitine and Co enzyme Q10 (COQ10) supplied to SMART Pro- medium on human sperm concentration, sperm morphology and chromatin structure during in vitro sperm activation. Eighty seven samples of semen of infertile and fertile men were randomly collected at the High Institute for Infertility Diagnosis and Assisted Reproductive Technologies/Al- Nahrain University. The mean age of the men was (32.034 \pm 0.57$)$ years with mean duration of infertility $(4.644 \pm 0.25)$ years. Seminal fluid analyses were done involving macroscopic and microscopic examinations according to WHO criteria. Swim-up technique was dependent for in vitro sperm activation; the washed samples were divided before using centrifugation swim-up technique into 3 groups: control group (G1)

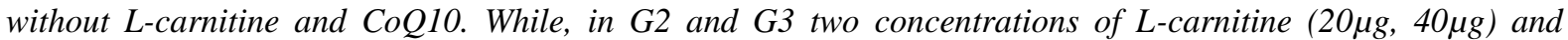
CoQ10 $(20 \mu g, 40 \mu g)$ were used respectively. Moreover, all groups within post-activation enriched with SMART-Pro media. The sperm concentration, sperm morphology and chromatin structure were evaluated after the addition of L-carnitine and Co Q10. The results showed a Significant $(P<0.05)$ differences in sperm parameters of post-activation when compared to the pre-activation. The results for all semen samples of post activation revealed a significant $(P<0.05)$ decrease in sperm concentration while, a significant $(P<0.05)$ increase in the percentage of normal sperm morphology as compared to pre-activation. The percentage of normal sperm morphology in $40 \mu \mathrm{g}(\mathrm{G} 3)$ group showed significant $(P<0.05)$ increase as compared to control group $(G 1)$ with no significant $(P>0.05)$ differences with $20 \mu \mathrm{g}(\mathrm{G} 2)$ group. However, $40 \mu \mathrm{g}(\mathrm{G} 3)$ group provides improvement in the percentages of normal sperm morphology as compared with 20 $\mu$ g (G2) group with no significant $(P>0.05)$ between $G 2$ and G3. A significant $(P<0.05)$ decrease in fragmented sperm DNA percentage of post activation was seen when compared with the pre-activation with non-significant $(P>0.05)$

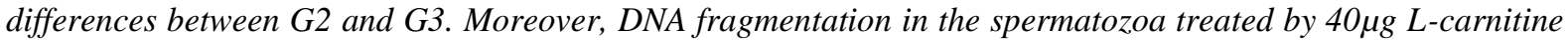
and CoQ10 was the lowest among post-activation groups. From the results of the study, it can be concluded that the addition of $40 \mu \mathrm{g}$ of L-carnitine and CoQ10 to washed sperms can improve sperm concentration and normal sperm morphology in vitro.
\end{abstract}

Keywords: L- Carnitine, COQ10, Sperm activation, Smart pro- medium

\section{Introduction}

Infertility is the inability of a sexually active non contraception couple to achieve pregnancy in one year [1]. Infertility can be due to the woman, the man, or both; primary or secondary. In primary infertility, the couples have never been able to conceive; while in secondary infertility there is difficulty in conceiving after having conceived ( either carried the pregnancy to term or had a miscarriage) [2].

A male factor is solely responsible in about $20 \%$ of infertile couples and contributory in another 30 $40 \%$, if a male infertility factor is present, it is almost always defined by the finding of an abnormal semen analysis for the assessment of male fertility [3]. Male infertility is due to the complete absence of sperm in the ejaculate and is relatively uncommon. Male sub fertility can be due to low numbers of sperm, a low percentage of sperm with effective progressive movement or abnormalities in the sperm-s ability to fertilize an egg [4]. Conventional semen analysis continues to be the only routine test to diagnose male factor infertility, although semen parameters have a limited power to predict spontaneous or assisted conception [5]. Semen analysis became the most important examination to be performed in the approach to the infertile couples [6]. Among these parameters, sperm morphology and motility are the best criteria for demonstrating the fertilization capacity of a male. Sperm morphology is a surrogate measure of the integrity of DNA packaging and the quality of spermatogenesis [7]. Currently, one of the four major sperm DNA fragmentation tests is the acridine orange test (AOT) [8]. AO test is a fluorescence staining called sperm chromatin structural assay (SCSA) which reflects sperm chromatin denaturation (single strand DNA vs. double strand DNA) [9]. 
L-carnitine play important role in sperm metabolism by providing readily available energy for use by spermatozoa, which positively affects sperm motility, maturation and the spermatogenic process [10]. Coenzyme Q10 is a non-enzymatic antioxidant that is related to low-density lipoproteins and protects against peroxidative damage. Since it is an energy-promoting agent, it also enhances sperm motility [11], and a major cellular antioxidant [12]. The aims of the study were to investigate the effect of different concentrations of (Lcarnitine and COQ10) supplied to culture medium on sperm concentration, sperm morphology and investigate the effects of (L-carnitine and COQ10 ) on the sperm DNA integrity for both fertile and infertile men.

\section{Materials and methods}

All samples of semen were collected after (3-5) days of abstinence directly in a clean, dry and sterile disposable Petri- dish by masturbation in a private and quite room adjacent to the semen analysis laboratory. Each Petri-dish was labeled with the person name, ageabstinence period and time of sample collection. The specimens were placed in an incubator at $37^{\circ} \mathrm{C}$ for 30 minutes to allow liquefaction [13]. The liquefied semen is then carefully mixed for few seconds to homogenize, and then subjected to both macroscopic and microscopic examinations within one hour from collection according to WHO manual (1999) [14]. Each sample was divided into three groups, after being centrifuged once with $2500 \mathrm{rpm}$ for 6 minutes, the supernatant was discarded and then gently $(1 \mathrm{~mL})$ of SMART Pro-medium was added only for the first group (G1), SMART Pro $+20 \mu \mathrm{g}$ of Lcarnitine and CoQ10 for the second group (G2) and SMART Pro + $40 \mu \mathrm{g}$ of L-carnitine and CoQ10 for the third group (G3). The samples placed in the incubator an inclined position and sperm parameters were read after 30 minutes. Furthermore, all samples from both fertile and infertile men were examined for SCSA to evaluate the percentage of DNA fragmentation by using AO test.The standard of WHO (2010) [1]is used to record details of the semen analysis results.

\section{Statistical analysis}

The data were statistically analyzed using SPSS/PC version 18 software (SPSS, Chicago).Sperm parameters pre- and post in vitro sperm activation and groups of infertile men were analyzed using complete randomized design (CRD) (one way ANOVA).

\section{Results}

Eighty seven infertile and fertile menwere involved in this study. The mean age of subjects was (32.034 \pm 0.57 )years old with the range (21-51) years. However, the mean duration of infertility was $(4.644 \pm$ $0.25)$ years with the range (1-12) years.The results of Macroscopic and microscopic parameters of semen analyses (SA) for subjects and sperm DNA fragmentations are shown in (Table1).In this table, the sperm concentration and normal sperm morphology (\%) were within normal ranges according to the criteria of WHO (2010) [1]. The results for all semen samples of post activation after using SMART- Pro medium supplemented with $20 \mu \mathrm{g}(\mathrm{G} 2)$ or $40 \mu \mathrm{g}(\mathrm{G} 3)$ of both L-carnitine andCoQ10) revealed a significant $(\mathrm{P}<0.05)$ decrease in sperm concentration ascompared to pre- activation with no significant $(\mathrm{P}>0.05)$ differences between $20 \mu \mathrm{g}(\mathrm{G} 2)$ and $40 \mu \mathrm{g}(\mathrm{G} 3)$ groups. While, a significant $(\mathrm{P}<0.05)$ increase in the percentage of normal sperm morphology in $40 \mu \mathrm{g}$ (G3) group as compared to control group (G1) with no significant ( $\mathrm{P}>0.05)$ differences with $20 \mu \mathrm{g}(\mathrm{G} 2)$ group (Table2).

Figure (1) showed the influence of SMART-Pro medium alone or acombination of two concentrations of L-carnitine and CoQ10 $(20 \mu \mathrm{g}$ or $40 \mu \mathrm{g})$ on sperm DNA fragmentation for all semen samples. With respect to sperm DNA fragmentation, a significant $(\mathrm{P}<0.05)$ decrease was observed between post and pre-activation.

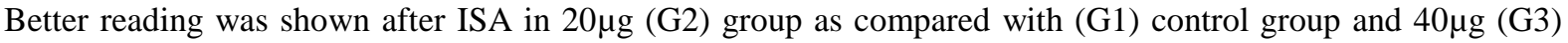
group with no significant $(\mathrm{P}>0.05)$ differences among the groups.

Table (1): Spermconcentration,morphology and DNA fragmentation (\%) for men involved in this study (Mean

\begin{tabular}{|c|c|c|}
\hline Parameters & Mean \pm S.E & $\begin{array}{c}\text { WHO(2010) } \\
\text { criteria }\end{array}$ \\
\hline Sperm concentration (Millions /mL) & $59.678 \pm 2.86$ & $\geq 15$ \\
\hline Normal sperm morphology (\%) & $35.092 \pm 0.97$ & $\geq 30 \%$ \\
& & $($ WHO,1992) \\
\hline Sperm DNA fragmentation (\%) & $24.363 \pm 2.40$ & $<30$ \\
\hline
\end{tabular}


Table (2):SMART-Pro medium enriched with two concentrations of L-carnitine and CoQ10 (20 $\mu \mathrm{g}$ and $40 \mu \mathrm{g})$ for all subjects (mean \pm S.E).

\begin{tabular}{|c|c|c|c|c|}
\hline \multirow[b]{2}{*}{ Parameters } & \multirow{2}{*}{ Pre-activation } & \multicolumn{3}{|c|}{ Post- activation } \\
\hline & & $\begin{array}{c}\text { Control } \\
\text { G1 }\end{array}$ & $\begin{array}{c}20 \mu \mathrm{g} \\
\text { L-carnitine and } \\
\text { CoQ10 } \\
\text { G2 }\end{array}$ & $\begin{array}{c}40 \mu \mathrm{g} \\
\text { L-carnitine and } \\
\text { CoQ10 } \\
\text { G340 } \mu \mathrm{g} \\
\text { G3 }\end{array}$ \\
\hline Sperm concentration millions $/ \mathrm{mL}$ & $\begin{array}{c}58.383 \text { a } \\
\pm 2.92\end{array}$ & $\begin{array}{c}31.062 \mathrm{~b} \\
\pm 2.69\end{array}$ & $\begin{array}{c}25.580 \mathrm{c} \\
\pm 2.53\end{array}$ & $\begin{array}{c}23.802 \mathrm{c} \\
\pm 2.55\end{array}$ \\
\hline Normal sperm morphology (\%) & $\begin{array}{c}34.790 \mathrm{c} \\
\pm 1.03\end{array}$ & $\begin{array}{c}91.643 \mathrm{~b} \\
\pm 1.58\end{array}$ & $\begin{array}{c}95.264 \mathrm{ab} \\
\pm 1.18\end{array}$ & $\begin{array}{c}97.716 \text { a } \\
\pm 0.36\end{array}$ \\
\hline
\end{tabular}

$*$ Number of subjects $=(87)$.

*Means with different superscripts within each row are significantly different $(\mathrm{P}<0.05)$.

*Means with similar superscripts within each row are non-significantly different $(\mathrm{P}>0.0 .5)$

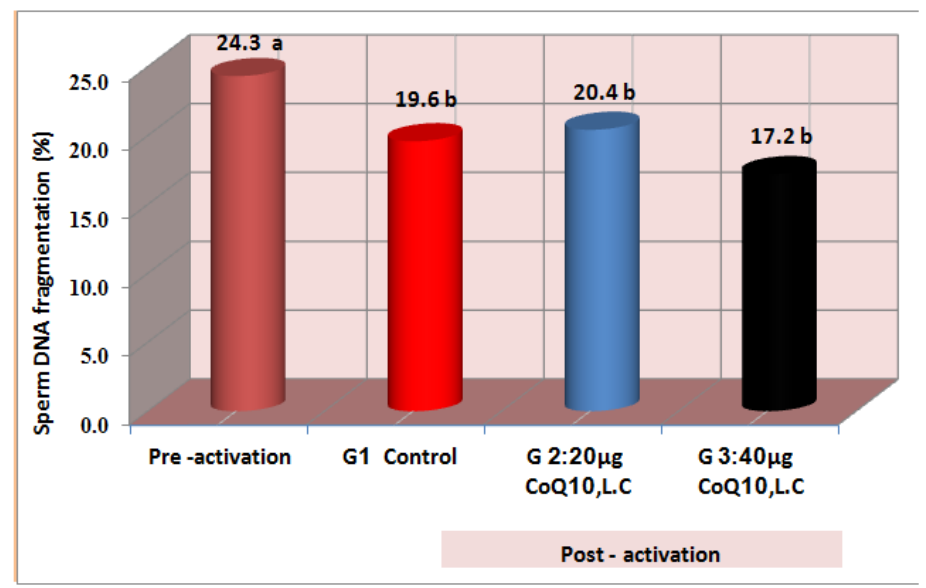

Figure (1) DNA fragmentation (\%) of pre- and post- in vitro sperm activation using SMART- Pro medium enriched with $(20 \mu \mathrm{g}, 40 \mu \mathrm{g})$ of L-carnitine and CoQ10 on sperm for all subjects.

*Means with different superscripts on each column are significantly different $(\mathrm{P}<0.05)$.

*Means with similar superscripts on each column are non-significantly different $(\mathrm{P}>0.05)$.

\section{Discussion}

In this study, a combination of the coenzyme Q10 (CoQ10) and L-carnitine (low concentration 20 $\mu \mathrm{g}$ and high concentration $40 \mu \mathrm{g}$ ) were supplemented to SMART-Pro medium to improve sperm parameters in vitro. A well improvement in the sperm parameters was showed for treated groups as compared to the pre activation group. Several factors have been participated for enhancing outcome of in vitro activation through sperm activity including presence of mitochondria with adequate numbers and normal activity [15], presence of ATP [16]. Normal and adequate gene expression for protein synthesis [17], integrity of plasma membrane [18], reduction level of ROS [19], presence of CoQ10 [20], L- carnitine contents and environment of culture media [21]. In the present study, centrifugation swim-up (technique was applied for in vitro sperm activation. Centrifuge swim-up was the most commonly methods used to separate the sperm fraction for use in ARTs [22]. Processing and isolating sperm from the whole semen specimen as highly motile spermatozoa have been tried with variable success before its use for ARTs [23].

Centrifugation swim-up technique was used in this study, the SUP technique relies on the ability of the motile spermatozoa to "swim up" into the culture medium, while slow and immotile sperm remain behind, along with other components in the semen pellet [24]. In the present study, sperm centrifugation was selected as a method for ISA depending of the results of the Shaaban (2007) [25]. With respect to sperm functional capacity, each laboratory should determine the centrifugation force and centrifugation time that are necessary to form a manageable recovery of sperm [26]. SMART- Pro medium which invented by Fakhrildin and Flayyih (2011) [27], medium was prepared based on Ringer solution supplemented with two essential additives Sodium lactate and/or pyruvate and human serum albumin (HSA) (5\% or 10\%). It was known that the albumin play a major 
role in physiology and metabolism of spermatozoa [28]. It is noticed in the current study that the use of in vitro SMART-Pro medium a significant $(\mathrm{P}<0.05)$ improvement in sperm parameters, as shown in (control) compared to pre-activation. Enhancement sperm parameters may be considered as normal response for sperm physiology after the removal of seminal plasma, pus cells and agglutinated spermatozoa using sperm preparation techniques. Furthermore, it was reported that only the active motile sperms will swim-up to the upper layer of culture medium in vitro human sperm activation $[29,30]$.

The results of the present study revealed a significant $(\mathrm{P}<0.05)$ decrease in the sperm concentration for control group compared to pre-activation. These results are considered normal during in vitro activation of human spermatozoa [31]. The reduction in the sperm concentration results from swim-up of only active motile sperm during ISA [30]. Centrifugation swim-up technique was used in the present work to remove most sperm with low motility and immotile sperm, with abnormal morphology, and agglutinated spermatozoa [31]. After activation, a significant improvement in the certain sperm function parameters was recorded. A significant $(\mathrm{P}<0.05)$ improvements in the percentages of sperm motility, progressive sperm motility, normal sperm morphology were noticed in both treated groups as compared to pre-activation groups. This may be related to the fast movement of normal spermatozoa from seminal plasma into layer of culture medium, and elicited from effect of some seminal plasma components like leukocytes, round cell and others that making the sperm out of stress factor and ROS production that responsible for DNA damage [32].

Moreover, when culture medium is supplemented with human serum albumin (HSA), further improvement in sperm parameters as a stimulator compared to control. Armstrong et al. (1998) [33] reviewed the beneficial effects of albumin and other protein on the motility and morphology of the recovered spermatozoa and mentioned the role of HSA as antioxidant. The cause of infertility in the infertile men with normal semen parameters could be related to abnormal sperm DNA [34]. Moreover, poor semen quality has been associated with an increase in the proportion of sperm with DNA fragmentation [35]. In the present study, the fragmentation of sperm DNA was considered an important procedure for male fertility and infertility diagnosis [36].

\section{References}

[1]. World Health Organization (WHO). (2010). WHO laboratory manual for the examination of human semen and semen-cervical mucus interaction. 5th edition. Cambridge: Cambridge University Press. pp:162-224.

[2]. Tan, Y.; and Bennett, M.J. (2007). The Australian \& New Zealand journal of obstetrics \&gynaecology, 47 (5): $406-409$.

[3]. Agarwal, H.S.; and Kulkarni, K.S. (2003). Efficacy and safety of semen in patient with oligospermia: an open clinical study. Indian J. Clin. Practice. 14 (2): 29-31.

[4]. Francavilla, F.; Sciarretta, F.; Sorgentone, S.; Necozione, S.; Santucci, R.; Barbonetti, A.; and Francavilla, S. (2009). Intra uterine insemination with or without mild ovarian stimulation in couples with male sub fertility due to oligo/asthenoand/ or teratozoospermia or anti sperm antibodies: a prospective cross-over trial. FertilSteril, 92(3): 1009 -1011.

[5]. Lewis, S.E.M. (2007). Is sperm evaluation useful in predicting human fertility? Reproduction, 134:1-11.

[6]. Karpuz, V.; Gokturk, A.; and Koyuturk, M. (2007). The Effect of Sperm Morphology and Motility on the Outcome of Intra cytoplasmic Sperm Injection. Marmara Med. J., 20 (2) ; 92-97.

[7]. Pacey, A.A. (2006). Is quality assurance in semen analysis still really necessary? A view from the andrology laboratory. Hum Reprod; 21:11059.

[8]. Lambardo, F.; Gandini, L.; Lenzi, A.; and Dondero, F. (2004). Anti sperm immunity in assisted reproduction. J ReprodImmunol. 62: $101-109$.

[9]. Tejada, R.I.; Mitchell, J.C.; Norman, A.; Marik, J.J.; and Friedman. (1984). A test for the practical evaluation of male fertility by acridine orange (AO) fluorescence. Fertil. Steril. 42 (1):87-91.

[10]. Matalliotakis, I.; Koumantaki, Y.; and Evageliou, A. (2000). L-carnitine levels in the seminal plasma of fertile and infertile men: correlation with sperm quality. Int J Fertil Women. 45: 236-240.

[11]. Lewin, A.; and Lavon, H. (1997). The effect of coenzyme Q10 on sperm motility and function. Mol Aspects Med, suppl. 18: S213219.

[12]. Bentinger, M.K. Brismar; and Dallner, G. (2007).The antioxidant role of coenzyme Q. Mitochondrion.7 Suppl:S41-S50.

[13]. NAFA and ESHRE. (2002). Nordic Association for Andrology and European Society of Human Reproduction and EmbryologySpecial Interest Group on Andrology. Manual on Basic Semen Analysis. 1-24.

[14]. World Health Organization (WHO). (1999).WHO laboratory manual for the examination of human semen and semen-cervical mucus interaction. 4th edition. Cambridge: Cambridge University Press.

[15]. Huntington, (2001). Study Group. A randomized, placebo controlled trial of coenzyme Q10 and re macemide in Huntington's Disease. Neurology. 57:397-404.

[16]. Miodrag, S.; Kirsten, W.; Valeri, Z.; Petra, S.; Katja, B.; and Eckhard, W. (1999). Coenzyme Q10 in Submicron-Sized Dispersion Improves Development, Hatching, Cell Proliferation, and Adenosine Tri phosphate Content of In Vitro-Produced Bovine Embryos. BIOL REPROD. 61, 541-547.

[17]. Crane, F.L. (2001). Biochemical functions of coenzyme Q10. J Am CollNutr 20: 591- 598.

[18]. Mizuno, K.; Tanaka, M.; Nozaki, S.; Mizuma, H.; Ataka, S.; Tahara, T.; Sugino, T.; Shirai, T.; Kajimoto, Y.; Kuratsune, H.; Kajimoto, O.; and Watanabe, Y. (2008). Anti fatigue effects of coenzyme Q10 during physical fatigue. Nutrition . 24: $293-299$.

[19]. Reynolds, J.A.; and Hand, S.C. (2004). Differences in isolated mitochondria are insufficient to account for respiratory depression during diapause in art emiafranciscana embryos. Physiol. Bio chem. Zool. 77, 366-377.

[20]. Levavasseur, F.; Miyadera, H.; Sirois, J.; Tremblay, M.L.; Kita, K.; and Shoubridge, E.;andHekimi, S. (2001). Ubiquinone is necessary for mouse embryonic development but is not essential for mitochondrial respiration. The Journal of Biological Chemistry, 276, 46160-46164.

[21]. Dallner, G. ; and Sindelar, P.J. (2000). Regulation of ubiquinone metabolism. Free RadicBiol Med. 29:285-294. 
[22]. Enciso, M.; Iglesias, M.; Galan, I.; Sarasa, J.; Gosalvez, A.; and Gosalvez, J. (2011). The ability of sperm selection techniques to remove single- or double-strand DNA damage. Asian J Androl. 13:764-768.

[23]. Cruz, R.I.; Kemmann, E.; Brandeis, V.T.; Becker, K.A.; Beck, M.; Beardsley, L.; and Shelden R.A. (1986). A prospective study intra uterine insemination of processed sperm from men with oligoasthenospermia in super ovulated women. FertilSteril. 46: 673677.

[24]. Alvarez, J.G; Lasso, J.L.; Blasco, L.; Nunez R.C.; Heyner, P.P.S.; and Caballero et al. (1993). Centrifugation of human spermatozoa induces sub lethal damage; separation of human spermatozoa from seminal plasma by a dextran swim-up procedure without centrifugation extends their motile lifetime. Hum Reprod. 8:1087-1092.

[25]. Shaaban, M.H. (2007). An in vitro human sperm activation study: using Hams F-12 medium and human serum albumin for infertile patients. MS.C. Thesis. Institute of embryo research and infertile treatment Al- Nahrainuniversity. Pp 102.

[26]. Isidori A.M.; Pozza, C.; Gianfrilli, D.; and Isidori, A. (2006). Medical treatment to improve sperm quality. Reprod Biomed Online, ; 12: $704-714$.

[27]. Fakhrildin, M.B.; and Flayyih, N.K. (2011). A new simple medium for in vitro sperm activation of asthenozoospermic patients using direct swim-up technique. Kufa Med. Journal. 14(1): 67-75.

[28]. King, R.S.; and Killian, G.J. (1994). Purification of bovine estrus associated protein and localization of binding on sperm. BiolReprod . 51: 34-42.

[29]. Mortimer, D. (2000). Sperm preparation methods. Andrology lab cancer. J. Androl. 21:334-340. 30

[30]. Henkel, R.; and Schill, W. (2003). Sperm preparation for ART. Repord Biol. And Endocrinol. 1:108-120

[31]. Harrison, R.A. (1976). Highly efficient method for washing mammalian spermatozoa. J. RepordFertil. 48:347-353.

[32]. Sharma, R.K.; Said, T.; and Agarwal A. (2004). Sperm DNA damage and its clinical relevance inassessing reproductive outcome. Asian. J. Androl. 6: 139-148.

[33]. Armstrong, J.S.; Raja sekaran, M.; Hellstrom, W.J.; and Sikka, S.C. (1998). Antioxidant potential of human serum albumin: role in the recovery of high quality human spermatozoa for assiste reproductive technology. Journal of Andrology, 19 (4): $412-419$.

[34]. Menezo, Y.J.; Hazout, A.; Panteix, G.; Robert, F.; Rollet, J.; and Cohen-Bacrie, P. (2007). Antioxidants to reduce sperm DNA fragmentation: an unexpected adverse effect. Reprod Biomed Online. 14: 418-421.

[35]. Irvine, D.S.; Twigg J.P.; Gordon, E.L.; Fulton, N.; Milne, P.A.; and Aitken, R.J. (2000). DNA integrity in human spermatozoa: relationships with semen quality. J Androl, Vol. 21, No. 1, pp.33-44.

[36]. Bungum, M. (2011). Sperm DNA integrity assessment: a new tool in diagnosis and treatment of fertility. P. 2012:1-6. 